\title{
Image-guided high-dose-rate brachytherapy of malignancies in various inner organs - technique, indications, and perspectives
}

\author{
Tina Bretschneider, MD', Jens Ricke, MD', Bernhard Gebaver, MD², Florian Streitparth, MD² \\ 'Department of Radiology, University of Magdeburg, Magdeburg, ${ }^{2}$ Department of Radiology. Charite - Universitätsmedizin Berlin, \\ Humboldt University, Berlin, Germany
}

\begin{abstract}
In the last few years, minimally invasive tumor ablation performed by interventional radiologists has gained increasing relevance in oncologic patient care. Limitations of thermal ablation techniques such as radiofrequency ablation (RFA), microwave ablation (MWA), and laser-induced thermotherapy (LITT), including large tumor size, cooling effects of adjacent vessels, and tumor location near thermosensitive structures, have led to the development of image-guided high-dose-rate (HDR) brachytherapy, especially for the treatment of liver malignancies. This article reviews technical properties of image-guided brachytherapy, indications and its current clinical role in multimodal cancer treatment. Furthermore, perspectives of this novel therapy option will be discussed.
\end{abstract}

J Contemp Brachytherapy 20l6; 8, 3: 253-263

DOI: $10.5114 / j c b .2016 .61068$

Key words: brachytherapy, HDR, image-guided, interventional oncology, radiofrequency ablation, thermal ablation.

\section{Purpose}

Potentially curative treatment is always the first-line approach in patients with early cancer. In patients with early liver cancer like hepatocellular carcinoma (HCC), thermal ablation allows excellent local tumor control, comparable to resection but less invasive, has fewer complications, and the loss of liver parenchyma is reduced [1]. For patients with advanced malignant disease and a shift in focus to palliation, minimally invasive ablation treatments have recently evolved into valid alternatives that supplement systemic drug regimens, and achieve at least temporary control of local tumor progression. Patients with oligometastatic disease may benefit from local treatment despite the impossibility to achieve true cure $[2,3]$.

Thermal ablation techniques such as radiofrequency ablation (RFA) and laser-induced thermotherapy (LITT) began to emerge as alternative treatment options to open surgery in the 1990s [4,5,6]. Microtherapeutic techniques have since developed continuously and, most recently, cryoablation [7], microwave ablation (MWA) $[8,9]$, and irreversible electroporation (IRE) [10] have been added to the growing armamentarium of minimally invasive treatment options [11]. All of these new minimally invasive approaches compete with the gold standard of open surgical resection in terms of effectiveness and tolerability.

Minimally invasive techniques based on hyperthermia have several limitations including a maximum tumor size of 3-3.5 cm, heat sink effects in the vicinity of large blood vessels, and the risk of causing cholestasis when treating lesions close to the thermosensitive bile ducts $[12,13,14,15]$. To overcome these limitations, image-guided interstitial high-dose-rate brachytherapy (HDRBT) was introduced into clinical practice by Ricke et al. in 2002 $[16,17]$. High-dose-rate brachytherapy has no restrictions with regard to the tumor size that can be treated; its therapeutic effect is not degraded by heat dissipation, and it can also be used to treat tumors in the vicinity of thermosensitive structures $[18,19]$. Unlike conventional external beam radiotherapy (EBRT), the therapeutic effect of HDRBT is not endangered by patient movement or respiratory excursion since the applicator is anchored directly within the tumor [20,21].

High-dose-rate brachytherapy is primarily used to achieve complete tumor elimination or tumor bulk reduction in patients with primary or secondary liver malignancies. Initial studies have reported the successful use of HDRBT in other organ systems including the lungs, lymph nodes, and the kidneys. These uses are also discussed in this educational article. 


\section{Interventional technique, treatment planning, and radiation properties}

The procedure consists of four major steps: 1) imageguided catheter implantation, 2) computer-based 3D treatment planning, 3) subsequent single fractionated high dose irradiation in afterloading technique, and 4) catheter removal and sealing of the puncture tract $[16,17,22]$.

As with all percutaneous interventions, HDRBT can only be performed when clotting function is not impaired (Quick > 50\%, thrombocytes > 50,000/ $\mu \mathrm{l}$, pTT < 50 s) and no infection is present. Following analgesia and sedation (intravenous [i.v.] fentanyl and midazolam), brachytherapy catheters are positioned using computed tomography (CT) or magnetic resonance imaging (MRI) fluoroscopy for guidance (Figure 1). After local anesthesia at the access site, the target lesion (primary tumor or metastasis) is punctured with a coaxial needle and a long $6 \mathrm{~F}$ angiographic introducer sheath with hydrophilic coating (Radiofocus, Terumo, Japan) is placed via a stiff angiographic guidewire (e.g., Amplatz, Boston Scientific, USA). The introducer sheath serves to insert the $6 \mathrm{~F}$ afterloading catheter (Primed, Halberstadt Medizintechnik GmbH, Halberstadt, Germany). One or multiple brachytherapy catheters will be inserted to cover the whole target volume. Positioning of the brachytherapy catheters inside the tumor volume is followed by a contrast-enhanced planning CT scan (iodine-based i.v. contrast agent [CA] at a volume of $80-120 \mathrm{ml}$ adjusted to body weight [BW]) or an MRI scan (gadolinium [Gd]-based i.v. CA; gadobutrol at $0.1 \mathrm{ml}$ per $\mathrm{kg} B W$ or Gd-EOB-DTPA at $0.1 \mathrm{ml}$ per $\mathrm{kg}$ $\mathrm{BW})$. It is recommended to perform the CT scan on a multislice spiral CT scanner or to acquire MRI during breathhold at a slice thickness of $5 \mathrm{~mm}$ or less. The CT or MRI dataset is used to determine the final catheter positions based on the tumor extent. Irradiation planning is performed by a physicist, an interventional radiologist and a radio-oncologist using a dedicated treatment planning workstation (BrachyVision, Varian Medical Systems, Charlottesville, VA, USA; or Oncentra, Nucletron, Elekta $A B$, Stockholm, Sweden) (Figures 1, 3-5). The catheter positions are entered as coordinates $(x, y, z)$ into the radiotherapy system. For each catheter, the distances to the tumor border are encoded using a system of reference points. High-dose-rate brachytherapy is performed using a ${ }^{192} \mathrm{Ir}$ source with $10 \mathrm{Ci}$ activity. Along the implanted brachytherapy catheters, the iridium source could be stopped for a defined time. The longer the source stops at a position, the larger the irradiated volume will be, so with multiple catheters even complex 3D volumes could be covered. Intratumoral HDRBT applicator positioning allows precise $3 \mathrm{D}$ radiation planning with application of
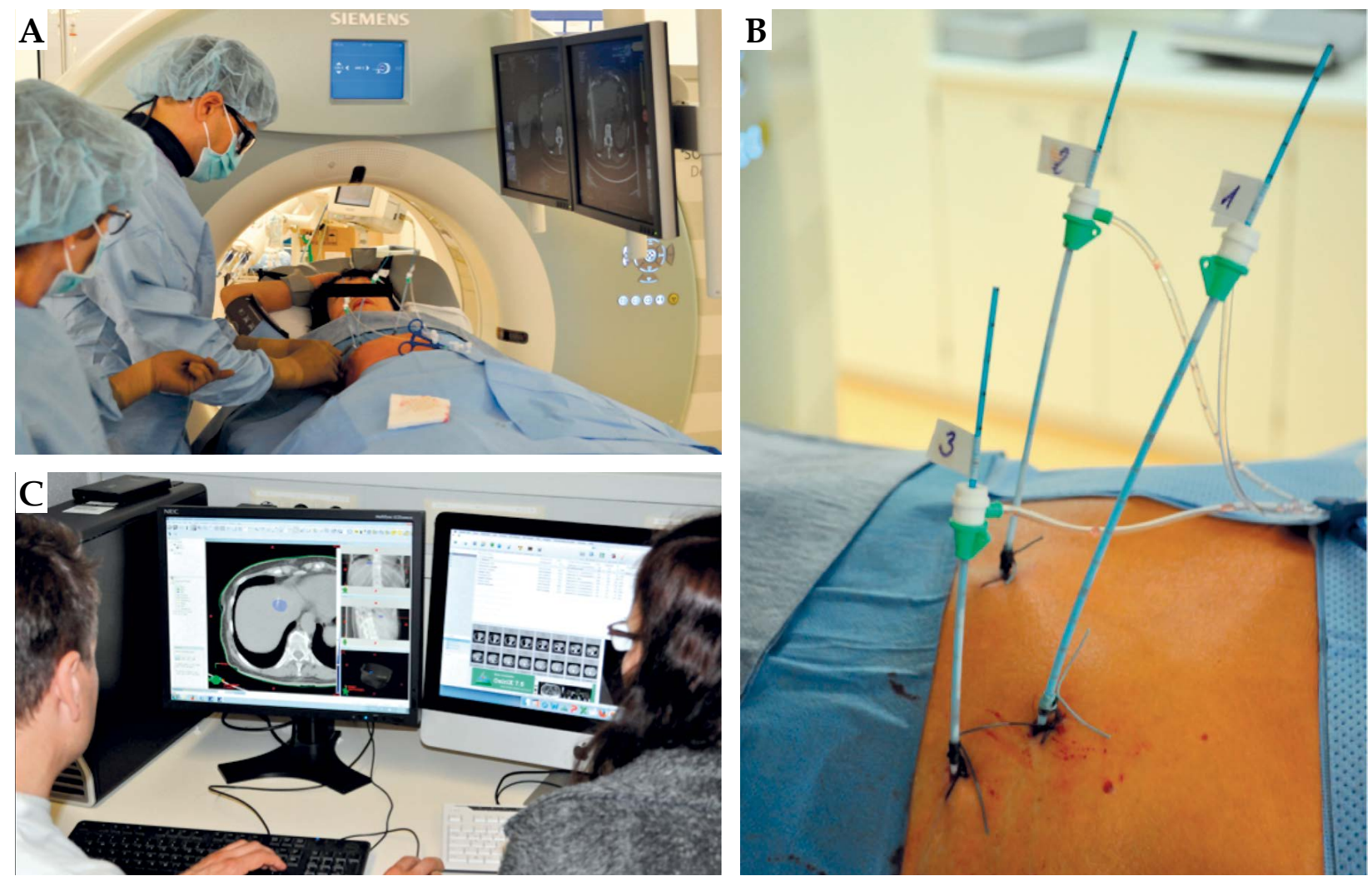

Fig. 1. 72-year-old female patient with metastatic colorectal cancer. A, B) Interventional setting in the computed tomography (CT) room with the patient placed in supine position. The interventionist and the assisting nurse are placing three applicators into three liver metastases. The CT-guided intervention can be monitored online via the in-room monitors on the right side in A. C) Irradiation planning is performed by an interventional radiologist and a physicist using a dedicated treatment planning workstation (BrachyVision, Varian Medical Systems, Charlottesville, VA, USA) 

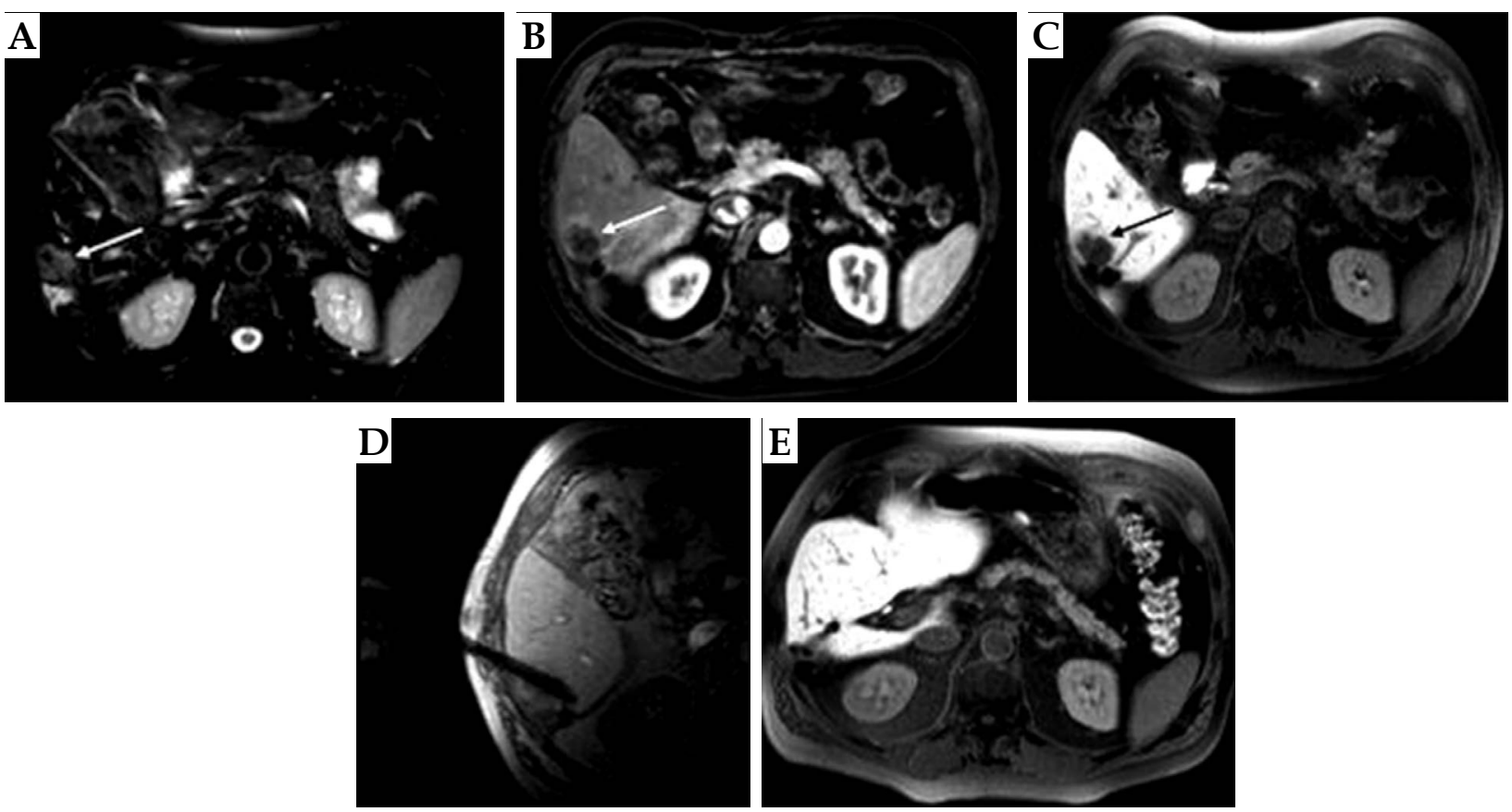

Fig. 2. 64-year-old female patient with metastatic colorectal cancer. A, B, C) Pre-therapeutic MRI of the liver using a liver-specific contrast agent (Primovist). Axial images (T2-weighted UTSE with FS; dynamic T1-weighted, arterial phase; T1-weighted WATS after contrast medium) show a recurrent lesion, approximately $3 \mathrm{~cm}$ in size (arrows), adjacent to the resection margin following atypical partial segmental resection (segments VI and V). D) Magnetic resonance imaging-guided positioning of a brachytherapy catheter in the liver metastasis in segment VI. E) Complete remission 85 months after high-dose-rate brachytherapy

a very high radiation dose to the target volume in a single fraction and a rapid drop in dose outside the target lesion. In this way, surrounding organs and structures are spared. The minimum target dose at the tumor border is $20 \mathrm{~Gy}$ and is planned as a single fraction. Higher doses within the tumor volume are acceptable and are not limited. High-dose-rate brachytherapy can also be used in tumors directly adjacent to the hepatic bifurcation or in tumors infiltrating central portal vein branches [16]. When a tumor is located in the vicinity of an organ at risk such as the stomach, the radiotherapy dose needs to be lowered accordingly. Radiation is usually applied for a duration of 10 to 40 minutes, depending on the clinical target volume (CTV). Data regarding a safe upper limit of irradiation duration are not available; the authors use an upper limit of approximately $60 \mathrm{~min}$ for application of clinical standard doses in very large target volumes. Tumor portions that do not receive enough radiation during the first HDRBT session may be targeted again in a second session. Ideally, the planned isodose should cover a few millimeters beyond the tumor border to ensure an adequate safety margin. Following radiation, the catheters are removed while administering gelfoam (liver, lymph nodes, kidney, adrenal gland) or fibrin glue (only for treatment of pulmonary lesions) to seal the puncture tract.

\section{Clinical indications and current state of research}

Therapeutic decisions in cancer patients should be made by a multidisciplinary team including surgeons, oncologists, hematologists and interventional radiolo- gists, and treatment should be tailored to the individual patient to ensure optimal outcome. Local tumor ablation may be one component of a multimodal treatment strategy, meaning that the newer local treatment options need to be integrated into existing regimens that may include systemic and/or local chemotherapy and combined surgical resection.

Several studies have investigated the effectiveness and safety of HDRBT. One advantage of HDRBT is that it can be used repeatedly to treat different target lesions in one organ or in different organs [23]. For patients with liver metastases, it has been shown that HDRBT is safe to use for repeated irradiation of the same site, for instance, in case of local recurrence [24].

\section{Primary and secondary liver tumors}

Hepatocellular carcinoma is commonly associated with cirrhosis, making it a complex condition that requires interdisciplinary assessment, since treatment planning must take not only on the tumor stage but also the patient's general condition and liver function into account. According to the Barcelona Clinic Liver Cancer (BCLC) staging system, image-guided tumor ablation is recommended for patients with early-stage HCC (single HCC or 3 nodules $<3 \mathrm{~cm}$ ), with the possibility of long-term cure and 5-year survival rate estimates ranging from $50 \%$ to $75 \%$ [25]. In very early $\mathrm{HCC}$ (BCLC 0 , solitary nodule $<2 \mathrm{~cm}$ ), tumor ablation is the accepted first-line therapeutic option [26,27]. Among different tumor ablation techniques, RFA is the most widely accepted modality. To achieve complete 

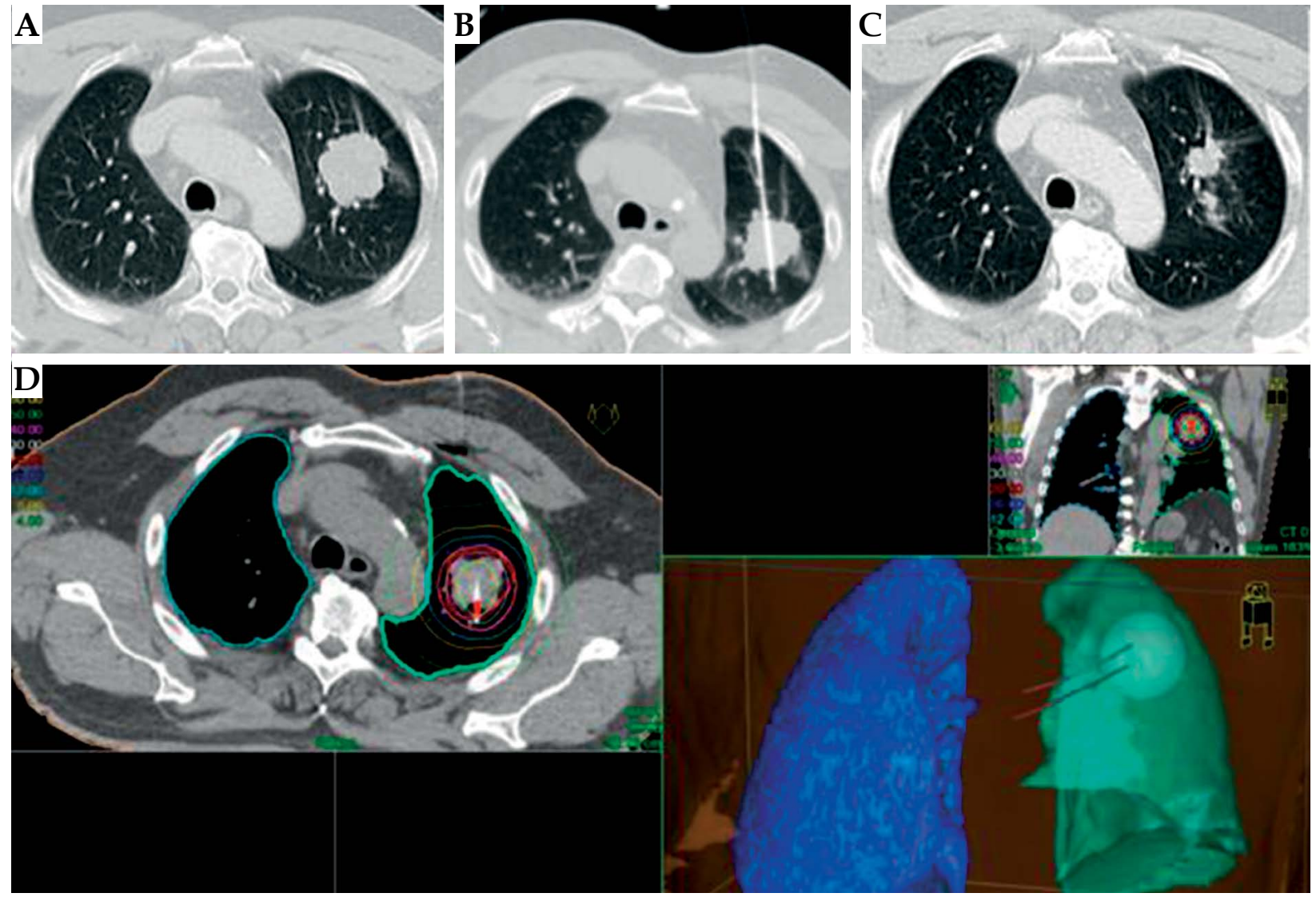

Fig. 3. A) 59-year-old patient with non-small-cell lung cancer (stage IV) in segments $1 / 2$ in the left upper lobe. The cancer has an axial diameter of approximately $6 \mathrm{~cm}$ and was first diagnosed in April 2012. Status: post resection of cerebral metastases and whole-brain radiotherapy, and palliative systemic chemotherapy with paclitaxel, carboplatin and Tarceva. B) Computed tomography (CT)-guided placement of a total of two brachytherapy catheters in the target volume. C) Two years after high-dose-rate brachytherapy, there is partial remission. D) 3-D radiotherapy plan with tumor-enclosing isodoses; target dose: 20 Gy, applied dose: 21.4 Gy

ablation and an adequate tumor-free margin with most of the currently available RF devices, the tumor should not exceed $3 \mathrm{~cm}$ in diameter [28]. When using RFA to treat $\mathrm{HCC}$ with a diameter of $3 \mathrm{~cm}$ to $5 \mathrm{~cm}$, it is recommended to perform arterial embolization prior to RFA to achieve adequate local tumor control rate [29].

Conventional percutaneous radiation of HCCs has some limitations, like the low radiation sensitivity of hepatocellular cancer cells and the radiation of liver parenchyma in these mostly cirrhotic patients. Although, stereotactic body radiotherapy (SBRT) could show statistically better local tumor control rates compared to RFA in HCC patient with tumors measuring 1.8 vs. $2.2 \mathrm{~cm}$ in diameter [30].

High-dose-rate brachytherapy allows a very high radiation dose to the tumor with a low dose to normal liver tumors and adherent organs. Preliminary studies with retrospective, matched-pair or prospective randomized designs demonstrated excellent local tumor control (LTC) rates of 93.3-96.1\%, and encouraging progression-free survival (PFS) and overall survival (OS) [16,18,31,32]. A prospective phase 2 study of HCC conducted by Mohnike et al. showed a high survival benefit compared to best supportive care (BSC): patients with a Cancer of the Liver Italian Program (CLIP) score of 2, had a median OS of 23 months in the brachytherapy group vs. 5 months in the control group; for patients with CLIP scores $\geq 3$, median OS was 18 vs. 4 months [31]. In the largest study, hitherto investigating patients with HCC treated by HDRBT, survival was not affected by patient age, gender or disease pattern, and lesion diameter (mean $>5 \mathrm{~cm}$ ) did not influence OS [32]. In a study evaluating HDRBT for HCC as a bridge to liver transplant, the results showed similar or even better outcome for HDRBT (histologically higher degree of necrosis and lower recurrence rate after liver transplant) than for transcatheter arterial chemoembolization (TACE) [33]. Hence, although TACE is the recommended first-line treatment for patients with intermediate stage HCC, the debate on alternative treatment strategies for patients with bulky and/or multifocal unresectable HCC is still open.

Approximately $60 \%$ of patients with advanced colorectal cancer are diagnosed with synchronous or metachronous liver metastases (CRLMs) (Figure 2) [34]. Depending on the extent and distribution of CRLMs and patient comorbidity, curative operation is not possible in approximately $80 \%$ of cases [35]. These patients may benefit from new systemic therapies including biological agents such as cetuximab and bevacizumab but also 

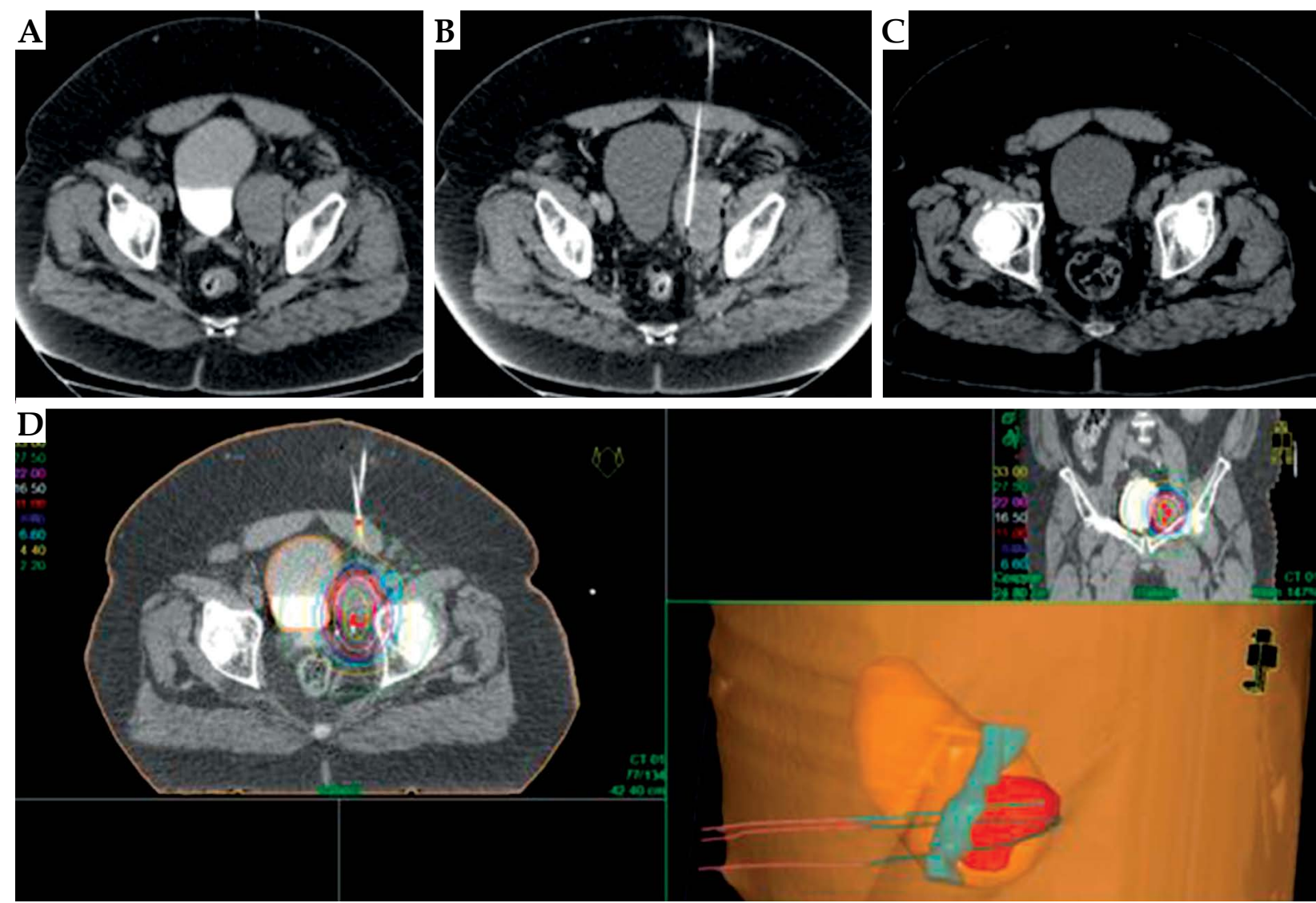

Fig. 4. A) 55-year-old patient with squamous cell CUP metastatic to the lymph nodes and bones. Status: post systemic chemotherapy with carboplatin and 5-FU. B) Computed tomography (CT)-guided positioning of a total of 3 catheters in the lesion in a left paravesical lymph node. C) Follow-up after 1 year shows partial remission of the left paravesical lymph node. D) 3D radiotherapy plan: hypofractionated brachytherapy on 2 successive days. $1^{\text {st }}$ fraction: target dose: 11 Gy, applied dose: 12.2 Gy; $2^{\text {nd }}$ fraction: target dose: $14 \mathrm{~Gy}$, applied dose: $14.5 \mathrm{~Gy}$

from percutaneous tumor ablation, which may even offer potentially curative treatment in selected patients [36]. Then, it is possible to combine local ablation with surgical resection or systemic regimens. The CLOCC study suggests that combined RFA and systemic therapy is superior to systemic therapy alone (PFS 16.8 vs. 9.9 months). Many studies have investigated RFA of CRLMs with variable success rates (local progression ranging from $11.9-42 \%)[4,37]$. In these studies, tumor size and location in the proximity of large blood vessels were shown to be the most important prognostic factors for the outcome of RFA in the treatment of liver metastases [37].

A retrospective study of Collettini et al. investigating CT-HDRBT in CRLMs found local tumor progression in $12.9 \%$ of cases and a median OS of 18 months [38]. Compared to HCC, the LTC rate in CRC liver metastases is reduced, probably because colorectal cancer tumor cells are more aggressive and less sensitive to radiation compared to hepatocellular tumor cells [32]. The authors concluded that a combination with other local and systemic therapies should be evaluated in patients with lesions $>4 \mathrm{~cm}$ in diameter, in which higher progression rates are expected [38]. In a prospective randomized phase II study of Ricke et al., repeated brachytherapy of CRLMs was found to be the most important prognostic factor even before salvage chemotherapies. The survival benefit was found to be greatest in patients treated with combined local brachytherapy and systemic salvage chemotherapy [23].

Other available data on the effect of the type of treatment on overall survival, also for metastases from other primaries such as breast cancer, are from single-arm prospective phase II studies or retrospective studies and are of limited value. In the treatment of liver metastases from breast cancers (BCLMs), two studies showed excellent LTC rates of $96.5-97.4 \%$ at 12 months $[39,40]$.

Use of CT-HDRBT for treatment of cholangiocarcinoma (CCC) or liver metastases from pancreatic adenocarcinoma, renal cell carcinoma, and gastric or gastroesophageal adenocarcinoma showed similar promising outcomes $[41,42,43]$. However, these reports have limited validity because they are based on retrospective studies and relative small patient populations.

For patients with unresectable and unablatable HCC, CRLMs, BCLMs, and other liver metastases in whom systemic and local therapies (chemotherapy, surgery, local ablative procedures) have been exhausted, ${ }^{90} \mathrm{Y}$ radioembolization (RE) and SBRT are alternative therapies $[44,45,46,47]$. 

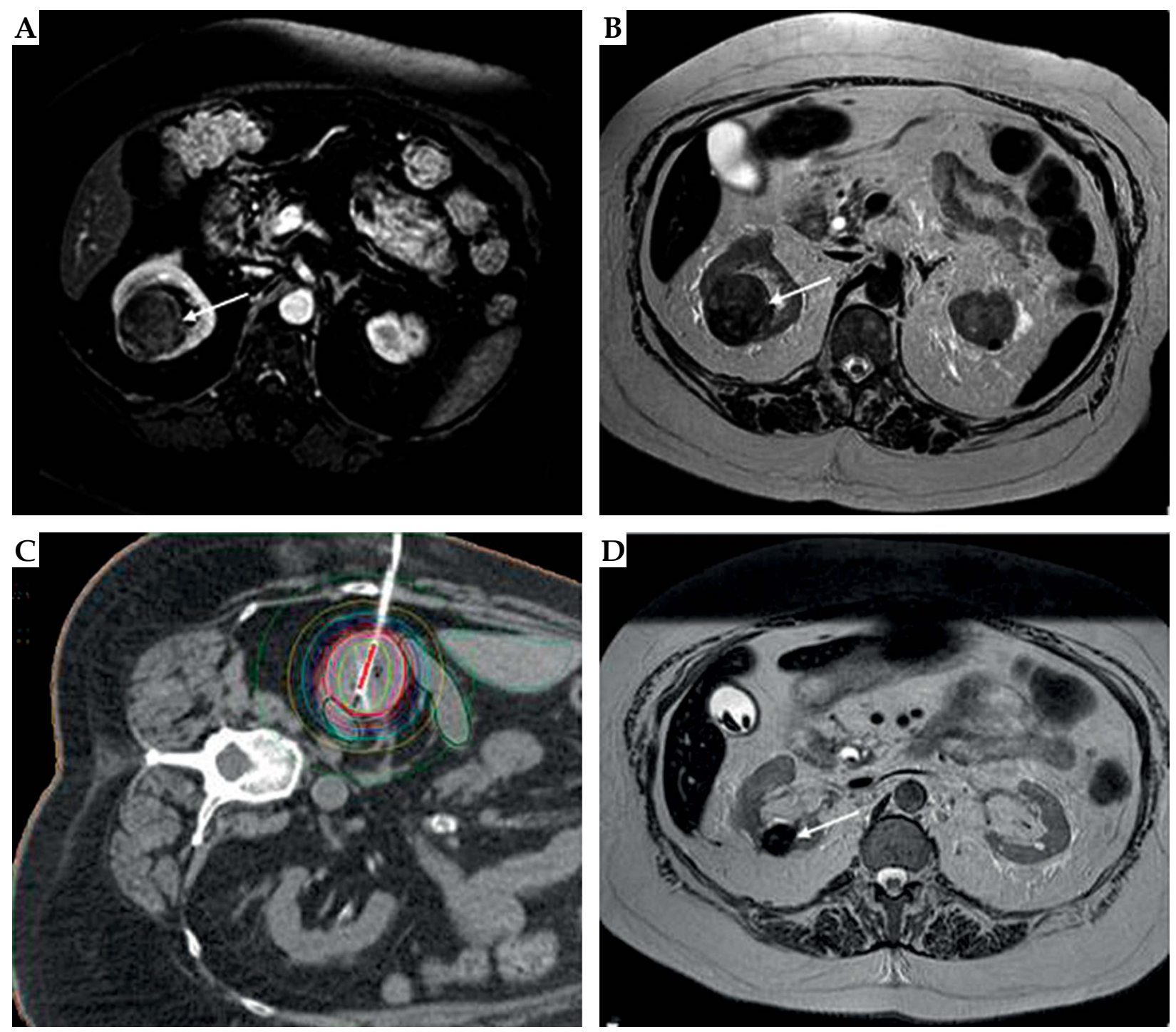

Fig. 5. 67-year-old female patient with bilateral renal cell carcinoma. A, B) Axial contrast-enhanced T1-weighted image with fat saturation and T2-weighted image without fat saturation show an inhomogeneous mass of low signal intensity, measuring up to approx. $4.2 \mathrm{~cm}$, in the upper to Middle third of the right Sidney. C) Patient in left lateral position for computed tomography (CT)-guided positioning of brachytherapy catheters in the tumor volume, tumor-enclosing isodoses. D) Follow-up 26 months after HDRBT shows clear partial remission

\section{Primary and secondary lung tumors}

In patients with early non-small-cell lung cancer (NSCLC) or pulmonary metastases, anatomical surgical resection is the therapeutic gold standard with 5- and 10 -year survival rates of $36 \%$ and $26 \%$, respectively. However, approximately two thirds of patients with pulmonary malignancy have poor lung function or serious comorbidity, precluding curative surgery [48]. New systemic chemotherapies have improved response rates in metastatic colorectal cancer but long-term survival remains poor [49]. Several minimally invasive therapeutic options are available for patients with inoperable lung tumors including SBRT (97.6\% LTC rate) [50], stereotactic radiosurgery with the CyberKnife (95\% LTC rate) [51], and imaging-guided tumor ablation [52]. The excellent
LTC rates reported for SBRT and CyberKnife treatment have for the most part been achieved in studies including patients with small tumors $(<5 \mathrm{~cm})$ and tumors in peripheral location.

Radiofrequency ablation is the most widely used thermal ablation technique [52,53]. Most centers use RFA in patients with a maximum of 5 pulmonary metastases and only for tumors that are smaller than $3.5 \mathrm{~cm}$. This means that only patients with stage IA or IB NSCLC can be treated by RFA [52]. Microwave ablation is a promising thermal ablation technique for the lungs, since it uses so called dielectric hysteresis to heat the tissue and thus, unlike RFA, does not depend on tissue conductivity or electrical current. This makes MWA a suitable technique for organs with higher tissue resistance such as the lung. Microwave-based systems are said to also allow abla- 
tion of larger tumor volumes and to be less susceptible to negative effects due to heat dissipation. In a most recent study, Egashira et al. reported an LTC of $94 \%$ for patients with a median lung tumor size of $12 \mathrm{~mm}$ (range 6-45 $\mathrm{mm})$ [54].

Compared with thermal ablation techniques, CTHDRBT allows successful treatment of markedly larger lung tumors $(>5 \mathrm{~cm})$ and of centrally located lesions using similar radiation doses as for the treatment of liver tumors (Figure 3). An exception are lesions in close vicinity to radiation-sensitive organs (esophagus, spinal cord). Initial LTC rates of $93.7-96.4 \%$ reported by Ricke et al., Peters et al., and Collettini et al. are very encouraging, especially in light of the fact that pulmonary lesions markedly larger than $3 \mathrm{~cm}$ in diameter were treated. Moreover, it was shown that local tumor progression could be treated successfully by repeated ablation $[55,56,57]$.

The combination of percutaneous tumor ablation and systemic chemotherapy might improve survival of patients with colorectal cancer and inoperable metastases. For example, Chua et al. report median overall survival of 21 months for RFA alone versus 59 months for RFA and chemotherapy [58].

\section{Extrahepatic extrapulmonary tumors}

Successful palliative treatment of tumors in various extrahepatic and extrapulmonary sites has also been described for HDR brachytherapy (Figure 4). In a study of Wieners et al., tumor locations included the hepatoduodenal ligament, mesentery, adrenal gland, mesogastrium, and local recurrences after rectal or pancreatic cancer. The median dose in the target volume was 11 Gy (4-18 Gy), resulting in $76.5 \%$ LTC and $47 \%$ progression-free survival at 6 months [59]. Treatment of lymph node metastases in para-aortic and periceliac location, and close to the hilum was found to be safe and effective, resulting in LTC in $80 \%$ of cases [60].

A recent study investigating treatment of malignant melanoma metastases in various locations, including the retroperitoneal lymph nodes besides the liver and lung, showed $90 \%$ LTC after a median follow-up of 5 months. Median overall survival was 8 months [61].

An overview of studies reflecting the current spectrum of image-guided HDR brachytherapy in different organ systems is provided in Table 1.

\section{Safety, complications, and their management}

Image-guided HDRBT in a single session has proven to be a safe procedure. The available data on the use of this technique in primary and secondary liver malignancies indicate that even considerable comorbidity or high age is not an absolute contraindication.

Brinkhaus et al. report that HDRBT of liver tumors does not impair hepatic function and shows high overall safety and favorable survival rates. They determined liver function in 69 patients after HDRBT. Liver function was assessed using conventional biochemical parameters and the LiMAx test based on 13C-methacetin kinetics. Liver enzymes and bilirubin normalized after 6 weeks.
There was no change in the LiMAx after the intervention and no patient developed radiation-induced liver disease (RILD) [63]. However, it has to be borne in mind that the risk of RILD is higher when liver tumor volumes are very large or in patients undergoing repeated brachytherapy. It is recommended to apply a dose of $\geq 10 \mathrm{~Gy}$ to a maximum of one third of the liver volume. A liver-protective regimen including enoxaparin, pentoxifylline, and ursodeoxycholic has been shown to significantly reduce the rate of RILD over an 8-week period following targeted radiotherapy [64]

Approximately, 2-3\% of patients undergoing liver interventions develop hemorrhage classified as grade 1 or 2 event according to Common Terminology Criteria for Adverse Events (CTCAE), and patients rarely require transfusion for this complication. In our patients, we observed bleeding requiring embolization for control (CTCAE grades 3-4) in approximately $1-2 \%$ of cases. All of these patients had cirrhosis with or without portal vein thrombosis. Therefore, we assume that the risk of bleeding is increased in patients with higher arterial blood flow due to cirrhosis. Patients with Child B cirrhosis had a 22-fold higher risk than patients with Child A disease [31]. We have observed that use of gelfoam instead of fibrin glue is very helpful, since filling of the tract with gelfoam during stepwise withdrawal of the catheter ensures a high degree of control.

In our experience, patients with a tumor in central location (e.g., central CCC) or patients after Whipple surgery and biliodigestive anastomosis have an increased risk of developing liver abscess or cholangitis. We use peri-interventional antibiosis in these patients (e.g., oral ciprofloxacin, $500 \mathrm{mg}, 1-0-1$, for 2 weeks). Overall, liver abscess formation occurs in only $1-2 \%$ of cases and is altogether rare.

Available experience suggests that the bile ducts are rarely affected by complications such as strictures and cholestasis. The dose tolerance of the large bile ducts in patients treated by HDRBT was investigated in recent retrospective analysis of 102 patients with different primary tumors (no cases of CCC) and liver metastases in central location. This analysis revealed a significant correlation between dose exposure and bile duct complications and a relatively high threshold (cutoff of $21.3 \mathrm{~Gy}$ per $1 \mathrm{ml}$ surface), suggesting that exposure of the large bile ducts is no limitation but must be considered in radiotherapy planning [65]. In contrast, there are dose limits that must be taken into account when treating lesions near the stomach or duodenum in order to prevent symptomatic ulceration. A prospective study included 33 patients with metastases near the stomach in liver segments II and III to investigate the gastric mucosal tolerance dose. This study identified a tolerance dose of $15.5 \mathrm{~Gy}$ per $1 \mathrm{ml}$ of organ surface for the clinical endpoint of endoscopically proven symptomatic gastric ulcer [66]. Proton pump inhibitors (PPI) are prescribed prophylactically when using interstitial radiotherapy for treating liver lesions in the vicinity of the stomach.

General symptoms such as nausea and vomiting are usually limited to the first day after the intervention, and occur in less than $10 \%$ of cases. Small asymptomatic pleu- 
Table 1. Overview of relevant original publications on image-guided high-dose-rate brachytherapy of malignancies in various inner organs, in chronological order. The table summarizes tumor entities investigated, number of patients and lesions included, lesion size, follow-up (meanand range), local progression, and median overall survival

\begin{tabular}{|c|c|c|c|c|c|c|}
\hline Author & Entity & $\begin{array}{l}\text { Patients/ } \\
\text { Tumors (n) }\end{array}$ & $\begin{array}{l}\text { Tumor } \\
\text { size }(\mathrm{cm})\end{array}$ & $\begin{array}{l}\text { Follow-up } \\
\text { (months) }\end{array}$ & $\begin{array}{c}\text { Local } \\
\text { progression (\%) }\end{array}$ & $\begin{array}{c}\text { Overall } \\
\text { survival } \\
\text { (months) }\end{array}$ \\
\hline Ricke et al. [16] & $\begin{array}{c}\text { Primary and secondary liver } \\
\text { malignancies }\end{array}$ & $37 / 38$ & $4.6(2.5-11)$ & $14(4-22)$ & 13 & NA \\
\hline Ricke et al. [17] & $\begin{array}{c}\text { Primary and secondary liver } \\
\text { malignancies }\end{array}$ & $20 / 20$ & $7.7(5.5-10.8)$ & $13(\mathrm{NA})$ & NA & 22 \\
\hline Mohnike et al. [31] & Hepatocellular carcinoma & $75 / 126$ & $4.4(1-15)$ & $\begin{array}{c}9.6(1.2- \\
58.5)\end{array}$ & 3.9 & 19.4 \\
\hline Collettini et al. [18] & Hepatocellular carcinoma $(\geq 5 \mathrm{~cm})$ & $35 / 35$ & $7.1(5-12)$ & $12.8(2-32)$ & 6.7 & 15.4 \\
\hline Collettini et al. [32] & Hepatocellular carcinoma & $98 / 192$ & $5(1.8-12)$ & $23.1(4-64)$ & 8.5 & 29.2 \\
\hline Schnapauff et al. [62] & Intrahepatic cholangiocarcinoma & $15 / 15$ & $5.2(1-18)$ & $18(1-27)$ & NA & 14 \\
\hline Ricke et al. [23] & Colorectal liver metastases & $73 / 199$ & $3.6(1-13.5)$ & $15.2(\mathrm{NA})$ & 25.1 & 27.9 \\
\hline Collettini et al. [38] & Colorectal liver metastases & $80 / 179$ & $2.8(8-10.7)$ & 16.9 (NA) & 12.9 & 18 \\
\hline Wieners et al. [39] & Breast cancer liver metastases & $41 / 115$ & $4.4(1-11)$ & $18(1-56)$ & 3.5 & NA \\
\hline Collettini et al. [40] & Breast cancer liver metastases & $37 / 80$ & $2.5(0.8-7.4)$ & $11.6(3-32)$ & 2.6 & $18(3-39)$ \\
\hline Wieners et al. [43] & $\begin{array}{c}\text { Pancreatic cancer liver } \\
\text { metastases }\end{array}$ & $20 / 49$ & $2.9(1.0-7.3)$ & $\begin{array}{c}13.7(1.4- \\
55.0)\end{array}$ & 10 & 8.6 \\
\hline Ricke et al. [57] & $\begin{array}{c}\text { Primary and secondary lung } \\
\text { malignancies }\end{array}$ & $15 / 30$ & $2(0.6-11)$ & $5(1-9)$ & 5 & NA \\
\hline Peters et al. [56] & $\begin{array}{c}\text { Primary and secondary lung } \\
\text { malignancies }\end{array}$ & $30 / 83$ & $2.5(0.6-11)$ & $9(1-21)$ & 3.6 & NA \\
\hline Collettini et al. [55] & $\begin{array}{c}\text { Primary and metastatic lung } \\
\text { malignancies }\end{array}$ & $22 / 33$ & $3.3(1-8.6)$ & $13.7(3-29)$ & 6.2 & NA \\
\hline Bretschneider et al. [61] & $\begin{array}{c}\text { Metastases of malignant } \\
\text { melanoma }\end{array}$ & $14 / 52$ & $1.5(0.7-10)$ & $5(1-11)$ & 10 & 8 \\
\hline Collettini et al. [60] & Lymph node metastases & $10 / 10$ & $3.6(1.2-6.7)$ & $13.2(4-20)$ & 20 & NA \\
\hline
\end{tabular}

Table 2. Organ-related dose limitations of highdose-rate brachytherapy at Charité, Berlin

\begin{tabular}{lcc} 
Organ & Dose in $1 \mathrm{cc}\left(\mathrm{D}_{1 \mathrm{cc}}\right)$ & Dose in $0.1 \mathrm{cc}\left(\mathrm{D}_{0.1 \mathrm{cc}}\right)$ \\
\hline Small intestine & $12 \mathrm{~Gy}$ & $15 \mathrm{~Gy}$ \\
\hline Colon & $12 \mathrm{~Gy}$ & $15 \mathrm{~Gy}$ \\
\hline Stomach & $12 \mathrm{~Gy}$ & $15 \mathrm{~Gy}$ \\
\hline Oesophagus & $15 \mathrm{~Gy}$ & $18 \mathrm{~Gy}$ \\
\hline Spinal canal & $10 \mathrm{~Gy}$ & $12 \mathrm{~Gy}$ \\
\hline Liver hilum & $18 \mathrm{~Gy}$ & $20 \mathrm{~Gy}$ \\
\hline Skin surface & $10 \mathrm{~Gy}$ & \\
\hline
\end{tabular}

$D_{0.1 c 0} D_{1 c c}-$ minimum dose to the most exposed $0.1 \mathrm{~cm}^{3}, 1 \mathrm{~cm}^{3}$ ral effusions are observed in $10-15 \%$ of patients undergoing HDRBT of liver lesions. Pleural effusion requiring treatment may occur when a larger portion of the diaphragm is exposed but this is a rare event. In a prospective phase 2 trial of 30 patients with 83 primary and secondary pulmonary malignancies treated by CT-guided HDRBT, there was one pneumothorax treated with an $8 \mathrm{~F}$ drain, while 6 patients developed small pneumothorax and did not require treatment [56]. This low complication rate is attributable to the fact that fibrin glue was used to seal the lateral aspect of the tract when removing the catheter, and that there is less mechanical damage to adjacent tissue compared with thermal ablation. Although the applicators for RFA and image-guided brachytherapy are similar in size, acute complications during and after the intervention such as pneumothorax appear to be more common when a thermal ablation technique is 
used. Published reports describe pneumothorax not requiring treatment in $30-50 \%$ of patients treated with RFA $[67,68,69]$.

Organ-related dose limitations of HDBRT at Charité, Berlin are shown in Table 2.

\section{New aspects and perspectives}

Besides the indications and studies discussed so far, there are a number of recent investigations aimed at expanding the spectrum and applications of HDRBT.

For patients with renal cell carcinoma (RCC), nephron-sparing surgery (NSS) is the gold standard, while percutaneous thermoablation, using RFA or MWA for instance, have evolved into additional curative treatment options. For stage T1a RCC $(<4 \mathrm{~cm})$ in peripheral or exophytic location and with enough distance from critical structures (calyceal system, ureter, vessels, intestine), treatment efficiency is nearly $100 \%$; in patients with T1b RCC ( $>4$ and $\leq 7 \mathrm{~cm}$ ), thermal ablation can be performed after prior transarterial embolization (TAE) [70]. Survival rates were found to be similar to NSS, while there were fewer complications and the initial decrease in glomerular filtration rate (GFR) was less pronounced [71]. However, ablation becomes difficult or impossible in many cases due to an unfavorable location, lesion size, or nearby critical structures.

In a preliminary study, the effectiveness and safety of HDRBT was evaluated in the treatment of 19 RCCs in 17 patients [72] (Figure 5). Catheters were positioned using CT or MRI fluoroscopy. In addition, imaging with CT or MRI, and renal function was assessed by MAG-3 renal scintigraphy as well as determination of serum creatinine and GFR to identify possible radiation-induced acute or chronic renal dysfunction. Renal function was assessed before and 3, 6, and 12 months after the intervention. The results indicate that, with application of a median dose of 46 Gy per milliliter of tumor volume, no patient suffered a significant loss of renal function over the 12 month follow-up period. Only two patients showed a temporary decrease in the tubular excretion rate in MAG-3 scintigraphy at 6 months with a complete return to normal in the further course. Local tumor control after a median follow-up of 9 months is $89.7 \%$ [72].

To further improve local control in patients with very large tumors $(>8 \mathrm{~cm})$, a study investigated the effect of hypofractionation on liver metastases from colorectal cancer [73]. In this study, the brachytherapy introducer sheaths were left in situ for 2 days and radiotherapy was delivered in 3 fractions of 10 Gy each. In this preliminary study, median OS was 17.5 months, and an LTC of $90 \%$ was achieved. No CTCAE grade III or IV adverse events were observed [73]. Other studies combining transarterial embolization with a radiosensitive drug (TACE) with HDBRT are already recruiting patients.

A most recent publication reports a joint interventional radiology and radiation oncology technique for achieving local control in challenging cases, for which other ablative techniques are contraindicated [74]. The lesions treated were in nontraditional locations such as pelvis, extremity, retroperitoneum, and head/neck in 18 patients with heavily pretreated malignancies. High-dose-rate brachythera- py was performed either in combination with EBRT or as monotherapy. The authors found a good one-year LTC of nearly $60 \%$ and a favorable toxicity profile.

While the routine imaging modality used to position brachytherapy catheters is CT fluoroscopy, it is also possible to position the catheters using MRI fluoroscopy in an open MRI system (1.0 T, Panorama, Phillips, The Netherlands). Compared with CT fluoroscopy, MRI fluoroscopy has the advantage of multiplanar imaging and better visualization, particularly of small liver lesions, when a hepatobiliary contrast agent is used (Gd-EOB-DTPA, Primovist ${ }^{\circledR}$, Bayer Healthcare, Germany) $[23,75]$.

A very recent study has analyzed and compared activity-based costs of HCC ablation using CT-guided HDRBT versus CT-guided RFA. Although HDRBT is a more complex procedure with more staff involved, it can be performed at lower costs per patient than RFA when treating more than 55 patients annually, mainly due to lower costs for disposables and a decreasing percentage of fixed costs with an increasing number of treatments [76].

In the future, additional prospective randomized controlled trials comparing HDRBT with standard treatments such as RFA and TACE are needed to generate evidence-based results for this relatively novel treatment method, and to define appropriate indications for various tumor entities and tumor stages.

\section{Conclusions}

Image-guided HDRBT is a safe and effective therapy option and has gained acceptance in integrated cancer treatment. It is most useful in a salvage situation in patients with large hepatic tumors, and the spectrum of applications in terms of tumor location, size, and number has been continuously expanded in recent years. Limitations such as cooling or lesion size are less of an issue when using HDRBT compared with thermal ablation techniques. In patients with advanced or metastatic malignancy, HDRBT allows satisfactory local tumor control and, therefore can temporarily halt disease progression, particularly in patients in whom chemotherapy fails. Future oncological treatment strategies should aim at optimally combining the different surgical, systemic, and interventional radiological treatment options to tailor treatment to each patient's individual needs. This requires patient-centered discussion and decision making by interdisciplinary tumor boards.

\section{Disclosure}

Authors report no conflict of interest.

\section{References}

1. Wang Y, Luo Q, Li Y et al. Radiofrequency ablation versus hepatic resection for small hepatocellular carcinomas: a meta-analysis of randomized and nonrandomized controlled trials. PLoS One 2014; 9: e84484.

2. Jones $R$, Stattner $S$, Sutton $P$ et al. Controversies in the oncosurgical management of liver limited stage IV colorectal cancer. Surg Oncol 2014; 23: 53-60.

3. Weichselbaum RR, Hellman S. Oligometastases revisited. Nat Rev Clin Oncol 2011; 8: 378-382. 
4. Solbiati L, Livraghi T, Goldberg S et al. Percutaneous radio-frequency ablation of hepatic metastases from colorectal cancer: long-term results in 117 patients. Radiology 2001; 221: 159-166.

5. Vogl T, Müller P, Mack M et al. Liver metastases: interventional therapeutic techniques and results, state of the art. Eur Radiol 1999; 9: 675-684.

6. Goldberg S, Gazelle G, Dawson S et al. Tissue ablation with radiofrequency: effect of probe size, gauge, duration, and temperature on lesion volume. Acad Radiol 1995; 2: 399-404.

7. Rong G, Bai W, Dong Z et al. Long-term outcomes of percutaneous cryoablation for patients with hepatocellular carcinoma within Milan criteria. PLoS One 2015; 10: e0123065.

8. Harari C, Magagna M, Bedoya M et al. Microwave Ablation: Comparison of Simultaneous and Sequential Activation of Multiple Antennas in Liver Model Systems. Radiology 2016; 278: 95-103.

9. Lubner M, Brace C, Ziemlewicz T et al. Microwave ablation of hepatic malignancy. Semin Intervent Radiol 2013; 30: 56-66.

10. Narayanan G. Irreversible Electroporation. Semin Intervent Radiol 2015; 32: 349-355.

11. Lencioni R, de Baere T, Martin R et al. Image-Guided Ablation of Malignant Liver Tumors: Recommendations for Clinical Validation of Novel Thermal and Non-Thermal Technologies - A Western Perspective. Liver Cancer 2015; 4: 208-214.

12. Curley S, Izzo F. Radiofrequency ablation of primary and metastatic hepatic malignancies. Int J Clin Oncol 2002; 7: 72-81.

13. Galandi D, Antes G. Radiofrequency thermal ablation versus other interventions for hepatocellular carcinoma. Cochrane Database Syst Rev 2004:CD003046.

14. Pech M, Werk M, Beck A et al. System continuity and energy distribution in laser-induced thermo therapy (LITT). Rofo 2002; 174: 754-760 [Article in German].

15. Rhim H. Percutaneous radiofrequency ablation therapy for patients with hepatocellular carcinoma during occlusion of hepatic blood flow: comparison with standard percutaneous radiofrequency ablation therapy. Cancer 2003; 98: 433-434; author reply 434-435.

16. Ricke J, Wust P, Stohlmann A et al. CT-guided interstitial brachytherapy of liver malignancies alone or in combination with thermal ablation: phase I-II results of a novel technique. Int J Radiat Oncol Biol Phys 2004; 58: 1496-1505.

17. Ricke J, Wust P, Wieners $G$ et al. Liver malignancies: CT-guided interstitial brachytherapy in patients with unfavorable lesions for thermal ablation. J Vasc Interv Radiol 2004; 15: $1279-1286$.

18. Collettini F, Schnapauff D, Poellinger A et al. Hepatocellular carcinoma: computed-tomography-guided high-dose-rate brachytherapy (CT-HDRBT) ablation of large $(5-7 \mathrm{~cm})$ and very large (>7 cm) tumours. Eur Radiol 2012; 22: 1101-1109.

19. Collettini F, Singh A, Schnapauff D et al. Computed-tomography-guided high-dose-rate brachytherapy (CT-HDRBT) ablation of metastases adjacent to the liver hilum. Eur J Radiol 2013; 82: e509-514.

20. Herfarth K, Debus J, Lohr F et al. Stereotactic single-dose radiation therapy of liver tumors: results of a phase I/II trial. J Clin Oncol 2001; 19: 164-170.

21. McGinn C, Ten Haken R, Ensminger W et al. Treatment of intrahepatic cancers with radiation doses based on a normal tissue complication probability model. J Clin Oncol 1998; 16: 2246-2252.

22. Ricke J, Seidensticker M, Ludemann L, et al. In vivo assessment of the tolerance dose of small liver volumes after single-fraction HDR irradiation. Int J Radiat Oncol Biol Phys 2005; 62: 776-784.

23. Ricke J, Mohnike K, Pech M et al. Local response and impact on survival after local ablation of liver metastases from col- orectal carcinoma by computed tomography-guided highdose-rate brachytherapy. Int J Radiat Oncol Biol Phys 2010; 78: 479-485.

24. Rühl R, Lüdemann L, Czarnecka A et al. Radiobiological restrictions and tolerance doses of repeated single-fraction HDR-irradiation of intersecting small liver volumes for recurrent hepatic metastases. Radiat Oncol 2010; 5: 44.

25. Lencioni R, Crocetti L. Local-regional treatment of hepatocellular carcinoma. Radiology 2012; 262: 43-58.

26. Livraghi T, Meloni F, Di Stasi M et al. Sustained complete response and complications rates after radiofrequency ablation of very early hepatocellular carcinoma in cirrhosis: Is resection still the treatment of choice? Hepatology 2008; 47: 82-89.

27. Cho Y, Kim J, Kim W et al. Hepatic resection versus radiofrequency ablation for very early stage hepatocellular carcinoma: a Markov model analysis. Hepatology 2010; 51: 1284-1290.

28. Crocetti L, de Baere T, Lencioni R. Quality improvement guidelines for radiofrequency ablation of liver tumours. Cardiovasc Intervent Radiol 2010; 33: 11-17.

29. Morimoto M, Numata K, Kondou M et al. Midterm outcomes in patients with intermediate-sized hepatocellular carcinoma: a randomized controlled trial for determining the efficacy of radiofrequency ablation combined with transcatheter arterial chemoembolization. Cancer 2010; 116: 5452-5460.

30. Wahl D, Stenmark M, Tao Y et al. Outcomes After Stereotactic Body Radiotherapy or Radiofrequency Ablation for Hepatocellular Carcinoma. J Clin Oncol 2016; 34: 452-459.

31. Mohnike K, Wieners G, Schwartz F et al. Computed tomography-guided high-dose-rate brachytherapy in hepatocellular carcinoma: safety, efficacy, and effect on survival. Int $J$ Radiat Oncol Biol Phys 2010; 78: 172-179.

32. Collettini F, Schreiber N, Schnapauff D et al. CT-guided highdose-rate brachytherapy of unresectable hepatocellular carcinoma. Strahlenther Onkol 2015; 191: 405-412.

33. Denecke T, Stelter L, Schnapauff D et al. CT-guided Interstitial Brachytherapy of Hepatocellular Carcinoma before Liver Transplantation: an Equivalent Alternative to Transarterial Chemoembolization? Eur Radiol 2015; 25: 2608-2616.

34. Geoghegan J, Scheele J. Treatment of colorectal liver metastases. Br J Surg 1999; 86: 158-169.

35. Garden O, Rees M, Poston G et al. Guidelines for resection of colorectal cancer liver metastases. Gut 2006; 55: 1-8.

36. Ruers T, Punt C, Van Coevorden F et al. Radiofrequency ablation combined with systemic treatment versus systemic treatment alone in patients with non-resectable colorectal liver metastases: a randomized EORTC Intergroup phase II study (EORTC 40004). Ann Oncol 2012; 23: 2619-2626.

37. Veltri A, Guarnieri T, Gazzera C et al. Long-term outcome of radiofrequency thermal ablation (RFA) of liver metastases from colorectal cancer (CRC): size as the leading prognostic factor for survival. Radiol Med 2012; 117: 1139-1151.

38. Collettini F, Lutter A, Schnapauff D et al. Unresectable colorectal liver metastases: percutaneous ablation using CT-guided high-dose-rate brachytherapy (CT-HDBRT). Rofo 2014; 186: 606-612.

39. Wieners G, Mohnike K, Peters N et al. Treatment of hepatic metastases of breast cancer with CT-guided interstitial brachytherapy - a phase II-study. Radiother Oncol 2011; 100: 314-339.

40. Collettini F, Golenia M, Schnapauff D et al. Percutaneous computed tomography-guided high-dose-rate brachytherapy ablation of breast cancer liver metastases: initial experience with 80 lesions. J Vasc Interv Radiol 2012; 23: 618-626.

41. Geisel D, Collettini F, Denecke T et al. Treatment for liver metastasis from renal cell carcinoma with computed-tomography-guided high-dose-rate brachytherapy (CT-HDRBT): a case series. World J Urol 2013; 31: 1525-1530. 
42. Geisel D, Denecke T, Collettini F et al. Treatment of hepatic metastases from gastric or gastroesophageal adenocarcinoma with computed tomography-guided high-dose-rate brachytherapy (CT-HDRBT). Anticancer Res 2012; 32: 54535458.

43. Wieners G, Schippers A, Collettini F et al. CT-guided highdose-rate brachytherapy in the interdisciplinary treatment of patients with liver metastases of pancreatic cancer. Hepatobiliary Pancreat Dis Int 2015; 14: 530-538.

44. Bujold A, Massey C, Kim J et al. Sequential phase I and II trials of stereotactic body radiotherapy for locally advanced hepatocellular carcinoma. J Clin Oncol 2013; 31: 1631-1639.

45. Salem R, Lewandowski R, Kulik L et al. Radioembolization results in longer time-to-progression and reduced toxicity compared with chemoembolization in patients with hepatocellular carcinoma. Gastroenterology 2011; 140: 497-507.e2.

46. Scorsetti M, Arcangeli S, Tozzi A et al. Is stereotactic body radiation therapy an attractive option for unresectable liver metastases? A preliminary report from a phase 2 trial. Int $\mathrm{J}$ Radiat Oncol Biol Phys 2013; 86: 336-342.

47. Jakobs T, Hoffmann R, Dehm K et al. Hepatic yttrium-90 radioembolization of chemotherapy-refractory colorectal cancer liver metastases. J Vasc Interv Radiol 2008; 19: 1187-1195.

48. Licker M, Spiliopoulos A, Frey J et al. Risk factors for early mortality and major complications following pneumonectomy for non-small cell carcinoma of the lung. Chest 2002; 121 1890-1897.

49. Douillard J, Cunningham D, Roth A et al. Irinotecan combined with fluorouracil compared with fluorouracil alone as first-line treatment for metastatic colorectal cancer: a multicentre randomized trial. Lancet 2000; 355: 1041-1047.

50. Timmerman R, Paulus R, Galvin J et al. Stereotactic body radiation therapy for inoperable early stage lung cancer. JAMA 2010; 303: 1070-1076.

51. Vahdat S, Oermann EK, Collins SP et al. CyberKnife radiosurgery for inoperable stage IA non-small cell lung cancer: 18F-fluorodeoxyglucose positron emission tomography/ computed tomography serial tumor response assessment. J Hematol Oncol 2010; 3: 6.

52. Dupuy DE. Image-guided thermal ablation of lung malignancies. Radiology 2011; 260: 633-655.

53. von Meyenfeldt E, Prevoo W, Peyrot D et al. Local progression after radiofrequency ablation for pulmonary metastases. Cancer 2011; 117: 3781-3787.

54. Egashira Y, Singh S, Bandula S et al. Percutaneous High-Energy Microwave Ablation for the Treatment of Pulmonary Tumors: A Retrospective Single-Center Experience. J Vasc Interv Radiol 2016; 27: 474-479.

55. Collettini F, Schnapauff D, Poellinger A et al. Percutaneous CT-guided high-dose brachytherapy (CT-HDRBT) ablation of primary and metastatic lung tumors in nonsurgical candidates. Rofo 2012; 184: 316-323 [Article in German].

56. Peters N, Wieners G, Pech M et al. CT-guided interstitial brachytherapy of primary and secondary lung malignancies: results of a prospective phase II trial. Strahlenther Onkol 2008 184: 296-301.

57. Ricke J, Wust P, Wieners G et al. CT-guided interstitial single-fraction brachytherapy of lung tumors: phase I results of a novel technique. Chest 2005; 127: 2237-2242.

58. Chua T, Thornbury K, Saxena A et al. Radiofrequency ablation as an adjunct to systemic chemotherapy for colorectal pulmonary metastases. Cancer 2010; 116: 2106-2114.

59. Wieners G, Pech M, Rudzinska M et al. CT-guided interstitial brachytherapy in the local treatment of extrahepatic, extrapulmonary secondary malignancies. Eur Radiol 2006; 16: 2586-2593.
60. Collettini F, Schippers A, Schnapauff D et al. Percutaneous ablation of lymph node metastases using CT-guided highdose-rate brachytherapy. Br J Radiol 2013; 86: 20130088.

61. Bretschneider T, Mohnike K, Hass P et al. Efficacy and safety of image-guided interstitial single fraction high-dose-rate brachytherapy in the management of metastatic malignant melanoma. J Contemp Brachytherapy 2015; 7: 154-160.

62. Schnapauff D, Denecke T, Grieser C et al. Computed tomography-guided interstitial HDR brachytherapy (CT-HDRBT) of the liver in patients with irresectable intrahepatic cholangiocarcinoma. Cardiovasc Intervent Radiol 2012; 35: 581-587.

63. Brinkhaus G, Lock JF, Malinowski M et al. CT-guided highdose-rate brachytherapy of liver tumours does not impair hepatic function and shows high overall safety and favorable survival rates. Ann Surg Oncol 2014; 21: 4284-4292.

64. Seidensticker M, Seidensticker R, Damm R et al. Prospective randomized trial of enoxaparin, pentoxifylline and ursodeoxycholic acid for prevention of radiation-induced liver toxicity. PLoS One 2014; 9: e112731.

65. Penzlin S, Hass P, Steffen I et al. Lokale Ablation von zentralen Lebertumoren mittels katheterbasierter Radiotherapie - Evaluation der Strahlentoleranz der großen Gallengänge. Rofo 2016; Supplement.

66. Streitparth F, Pech M, Bohmig M et al. In vivo assessment of the gastric mucosal tolerance dose after single fraction, small volume irradiation of liver malignancies by computed tomography-guided, high-dose-rate brachytherapy. Int J Radiat Oncol Biol Phys 2006; 65: 1479-1486.

67. Hosten N, Stier A, Weigel C et al. Laser-induced thermotherapy (LITT) of lung metastases: description of a miniaturized applicator, optimization, and initial treatment of patients. Rofo 2003; 175: 393-400.

68. King J, Glenn D, Clark W et al. Percutaneous radiofrequency ablation of pulmonary metastases in patients with colorectal cancer. Br J Surg 2004; 91: 217-223.

69. Steinke K, Sewell PE, Dupuy D et al. Pulmonary radiofrequency ablation--an international study survey. Anticancer Res 2004; 24: 339-343.

70. Takaki H, Soga N, Kanda $\mathrm{H}$ et al. Radiofrequency ablation versus radical nephrectomy: clinical outcomes for stage T1b renal cell carcinoma. Radiology 2014; 270: 292-299.

71. Guan W, Bai J, Liu J et al. Microwave ablation versus partial nephrectomy for small renal tumors: intermediate-term results. J Surg Oncol 2012; 106: 316-321.

72. Bretschneider T, Tienken C, Mohnike K et al. Bildgesteuerte HDR-Brachytherapie von Nierenmalignomen und nierennah gelegenen Metastasen: Evaluation der Nierentoleranzdosis. Fortschr Röntgenstr 2013; 185: VO319-4.

73. Hass P, Röllich B, Bretschneider T et al. Durchführbarkeit und Verträglichkeit einer hypofraktionierten interstitiellen Brachytherapie von sehr großen oder oligonodulären Lebermetastasen kolorektaler Karzinome. Strahlentherapie und Onkologie 2013;189: Supplement.

74. Kishan A, Lee E, McWilliams J et al. Image-guided highdose-rate brachytherapy: preliminary outcomes and toxicity of a joint interventional radiology and radiation oncology technique for achieving local control in challenging cases. J Contemp Brachytherapy 2015; 7: 327-335.

75. Fischbach F, Thormann M, Seidensticker M et al. Assessment of fast dynamic imaging and the use of Gd-EOB-DTPA for MR-guided liver interventions. J Magn Reson Imaging 2011; 34: 874-879.

76. Schnapauff D, Collettini F, Steffen I et al. Activity-based cost analysis of hepatic tumor ablation using CT-guided highdose rate brachytherapy or CT-guided radiofrequency ablation in hepatocellular carcinoma. Radiat Oncol 2016; 11: 26. 